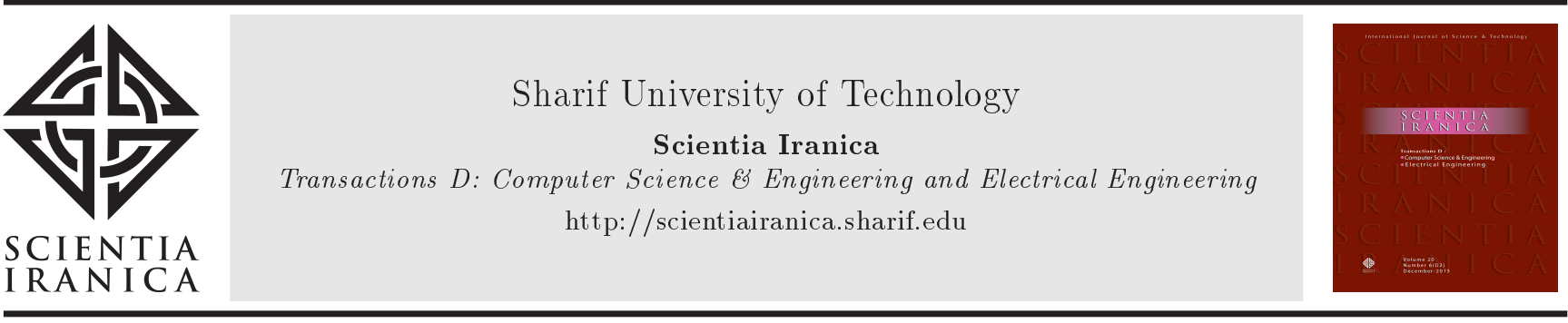

\title{
A holistic day-ahead distributed energy management approach: Equilibrium selection for customers' game
}

\author{
A. Shojaei Berjouei, M. Moallem*, and M.H. Manshaei \\ Department of Electrical and Computer, Isfahan University of Technology, Isfahan, 84156-83111, Iran.
}

Received 11 August 2017; received in revised form 26 May 2018; accepted 11 August 2018

\author{
KEYWORDS \\ Smart grid; \\ Energy management; \\ Generalized Nash \\ game; \\ Load factor; \\ Proximal Tikhonov \\ regularization \\ algorithm.
}

\begin{abstract}
$\overline{\text { Abstract. In this paper, a new holistic day-ahead distributed energy management }}$ approach with desired equilibrium selection capability in a smart distribution grid is proposed. The interaction between customers and the distribution company was modeled as a single-leader multiple-follower Stackelberg game. The interaction among customers was modeled as a non-cooperative generalized Nash game as they were faced with a common constraint. Customers held the average of the aggregate loads in the appropriate domain to reshape it and improve the Load Factor (LF). The strategy of the distribution company was day-ahead energy pricing through maximizing profit, which was formulated as a stochastic conditional value in risk optimization to consider the uncertainty in price of electricity in the wholesale market. Strategies of the customers were based on hourly consumption of deferrable loads and scheduled charge/discharge rates of energy storage devices in response to price. The generalized Nash game had multiple equilibria. Hence, the distributed proximal Tikhonov regularization algorithm is proposed here to achieve the desired equilibrium. The simulation results validated the performance of the proposed algorithm with $31.46 \%$ increase in the LF besides $45.89 \%$ and $14.23 \%$ reduction in the maximum aggregate demand and aggregate billing cost, respectively.
\end{abstract}

(C) 2020 Sharif University of Technology. All rights reserved.

\section{Introduction}

The significant growth in the electricity demand, limited fossil fuel resources, and increase in greenhouse gasses have led the researchers to run studies on new Demand-Side Management (DSM) programs. DSM improves energy consumption of the system at the side of the consumer [1]. DSM includes enhancing energy efficiency [2,3], smart energy pricing [4], demand response programs [5], optimal deployment of distributed energy resources [6], and energy storage devices at the side of the consumer [7].

\footnotetext{
*. Corresponding author. Tel.: +98313 3915381;

Fax: +983113912451

E-mail address: moallem@cc.iut.ac.ir (M. Moallem)
}

Due to the smart control, smart measurement, and two-way communication capability of the smart grid, DSM can be employed effectively. These advantages allow an intelligent interaction between different entities of distribution grid by providing and exchanging necessary information. This network develops smart pricing schemes, particularly real-time pricing policy, in implementing DSM in an effective and efficient manner.

DSM can be employed in the centralized and decentralized approaches. In the centralized approach, a central controller, basically at utility side, should collect all necessary information. This information includes many parameters and constraints from all agents of the smart grid to calculate optimal consumption schedule of customers. This approach is often impractical due to enormous computation and communication saturation in addition to privacy dis- 
closure. To overcome these drawbacks, decentralized DSM methods are developed and adopted.

Many researchers have proposed distributed automated algorithmic DSM programs for implementation in the smart distribution grid. Most of them have employed game theory as an analytical tool to model the optimization problem (e.g. [6,8-13]). The major part of these studies involves customers who anticipate prices and locally runs an iterative best-response algorithm to solve a distributed optimization problem. The results of these algorithms reveal the optimal energy consumption profile of every customer.

Implementation of efficient DSM programs depends on consumer and utility sides. Thus, in the other group of papers, the customers and distribution company DISCO are decision makers in the DSM game. These studies model the interaction of the customers and utility company as a Stackelberg game. Employing the Stackelberg game for modeling the interaction can be seen in $[4,5,7,14,15]$, and Maharjan et al. [15]. In such approach, customers are price takers who accept the price as a fixed parameter.

In most studies, the energy management problem is modeled as a game with a unique equilibrium; but, depending on the structure of the optimization problem as well as interaction and behavior of the players, the energy management game may have multiple equilibria. In these models, applying the distributed best-response algorithm is not responsive. However, recently, promising advances in solution computation for monotone games have been made by Scutari et al. $[16,17]$, which motivated us to develop a monotone energy management approach in a smart distribution grid.

In this article, new holistic day-ahead distributed energy scheduling for a smart distribution grid is proposed. Our proposed energy management mechanism addresses the interaction between utility grid and smart customers in a local distribution electricity market. DISCO formulates and solves an optimization-based problem of Conditional Value at Risk (CVaR) to obtain the optimal day-ahead hourly price of energy in the presence of uncertainty in the electricity price in the wholesale market. This optimal price is announced to residential customers through a two-way communication facility.

The price-taker customers are equipped with an Energy Management System (EMS) that receives the price and minimizes electricity bill to the customer while maximizing their utility. Moreover, EMSs try to meet the shared constraint together, leading to improvement in the appropriate shaping of the aggregate load, efficient utilization of the grid, and decrease in the operation grid cost. Here, customers limit the Average Aggregate Load (AAL) in the specified domain; therefore, the Load Factor (LF) can be settled in an appropriate range. Under such circumstance, a global constraint is satisfied that couples the strategy spaces of customers with each other.

This holistic energy management is modeled as a single-leader multiple-follower Stackelberg game, in which DISCO acts as the leader and customers are the followers. The interaction among customers can be modeled as a non-cooperative Generalized Nash (GN) game [16] because of their self-interested behavior and the shared constraint. The existence of a solution to the monotone game among customers, i.e., the GN equilibrium, is assessed here. A distributed Proximal Tikhonov Regularization Algorithm (PTRA) is applied to reaching the GN equilibrium, which is the essential section of the proposed algorithm for achieving the Stackelberg Equilibrium (SE). The convergence conditions of the newly proposed energy management algorithm are subject to the Variational Inequality (VI) theory. This algorithm selects the equilibrium that decreases the maximum aggregate demand and improves the LF at the global constraint among multiple equilibria of the GN game.

The paper is organized as follows. In Section 2, the model of the system is presented and the optimization problem is formulated. Then, the proper game framework, which captures the energy management interaction, and the solution to the game are introduced and discussed in Section 3. The distributed algorithm for achieving SE is proposed in Section 4. In Section 5, the implementation issues of the proposed energy management mechanism are discussed. Numerical results and performance evaluation of the proposed energy management algorithm are presented in Section 6. Finally, Section 7 concludes the paper.

\section{System modeling and formulation}

An automatic day-ahead energy management approach in a smart distribution system consisting of DISCO and its smart customers is studied. Every customer is assumed to have renewable energy resources, elastic and inelastic loads, and energy storage devices. The sets of smart homes and operation time slots are represented by $\mathcal{N} \triangleq\{1, \ldots, N\}$ and $\mathcal{T} \triangleq\{1, \ldots, T\}$, respectively; it is assumed that $T=24$.

Every customer is equipped with an EMS connected to both the electrical feeder and communication link, as shown in Figure 1. The EMSs can share realtime information through bidirectional communications with each other and with DISCO. EMS schedules energy consumption of a smart home in response to the price information.

DISCO sends hourly prices to its customers. Based on the price vector, every EMS decides on its daily load profile, $l_{n}=\left[l_{n}^{1}, \ldots, l_{n}^{t}, \ldots, l_{n}^{T}\right]$, through stor- 


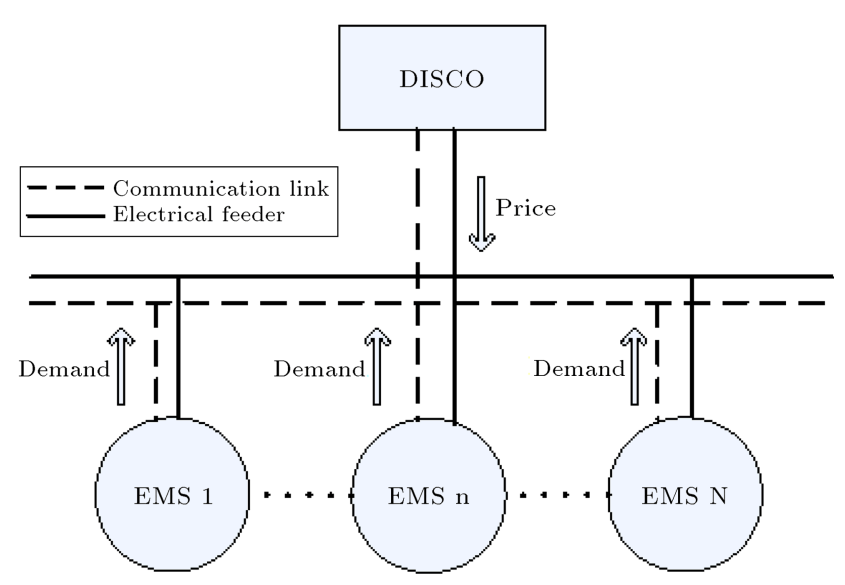

Figure 1. Smart distribution grid.

age devices and flexible appliances scheduling. Then, every EMS sends the load profile back to DISCO. Consequently, DISCO will compute the new optimal price, again, based on the current best-response strategy of customers. This procedure is repeated until an equilibrium is achieved.

In this smart distribution grid, it is assumed that the customers agree with DISCO on meeting the global constraint, AAL, voluntarily or through incentive contract. By adjusting the AAL parameter, LF increases and proper aggregate load shaping can be achieved. LF is calculated as follows:

$$
L F=\frac{L_{a v e}}{L_{\text {Peak }}}=\frac{\sum_{t \in \mathcal{T}} l^{t}}{T \max _{t \in \mathcal{T}} l^{t}},
$$

where $l^{t}=\sum_{n \in \mathcal{N}} l_{n}^{t}$ is the aggregate load of the customers at the time slot $t$. It is also assumed that every customer follows its committed day-ahead consumption profile, strictly. In the following, we address the objective models for DISCO and smart homes.

\subsection{Objective model of the DISCO}

DISCO should meet the electricity demanded by its clients in the wholesale market in all time horizons of the operations. DISCO can learn the demand behavior of its customers through different demand response programs with the help of amenities and facilities along with the smart grid.

DISCO predicts day-ahead energy prices, $\boldsymbol{\pi}$, in the wholesale day-ahead market based on the historical data. Then, by maximizing its profit, DISCO derives the optimal day-ahead sale price, $\boldsymbol{\rho}$, and sends it to the customers. Accordingly, DISCO is exposed to the following optimization problem:

$$
\operatorname{Max}_{\boldsymbol{\rho} \in \mathcal{P}} f_{D}(\boldsymbol{\rho}, \boldsymbol{l})=(1-\mu) \sum_{t \in \mathcal{T}} \mathbb{E}\left\{\left(\rho^{t}-\pi^{t}\right) l^{t}\right\}
$$

$$
\begin{aligned}
& +\mu C \operatorname{VaR}_{\alpha}\left\{\sum_{t \in \mathcal{T}}\left(\rho^{t}-\pi^{t}\right) l^{t}\right\}, \\
& \mathcal{P} \triangleq\left\{\left(\rho^{t}\right)_{t=1}^{T}: \rho_{\min }^{t} \leq \rho^{t} \leq \rho_{\max }^{t},\right. \\
& \left.1 / T \sum_{t \in \mathcal{T}} \rho^{t} \leq \rho_{\text {ave }}\right\}
\end{aligned}
$$

where $C V a R_{\alpha}$ is the expected profit in $(1-\alpha) \times 100 \%$ worst scenarios and $\alpha \in[0,1]$ is a confidence level. $\mu$ is a weighting factor that determines how much DISCO is a risk-neutral or risk-averse agent. In fact, DISCO uses CVaR optimization framework to handle the uncertainty in the prediction of the day-ahead wholesale market electricity prices and guarantee a certain amount of profit.

DISCO chooses an optimal price from the strategy set $\mathcal{P}$ based on the best response of the customers. Due to the grid configuration limitations, the load should not exceed a certain amount and the minimum dayahead price $\rho_{\text {min }}^{t}$ should be specified. Furthermore, the price should not be set too high until the minimum load $l_{\text {min }}^{t}$ is met. This fact indicates that the price is restricted by an upper bound, which is $\rho_{\max }^{t}$. To alleviate the market power of DISCO, an upper limit, $\rho_{\text {ave }}$, is imposed on the average price by the system regulator. By such a scheme, consumption behavior of the customers and DISCO activity can be regulated. Using Eq. (2) is difficult in evaluating the continuous profit distribution. To deal with this problem, Monte Carlo sampling is utilized to draw K i.i.d. samples from the profit distribution so that Eq. (2) can be rewritten in a tractable form as:

$$
\begin{aligned}
\operatorname{Max}_{\rho, c, z} & f_{D}(\boldsymbol{\rho}, \boldsymbol{l}) \\
= & \sum_{t \in \mathcal{T}}\left[(1-\mu)\left\{\left(\rho^{t}-\frac{1}{K} \sum_{k=1}^{K}\left(\pi_{k}^{t}\right)\right) l^{t}\right\}\right. \\
& \left.+\mu\left\{c^{t}-\frac{1}{1-\alpha} \frac{1}{K} \sum_{k=1}^{K} z_{k}^{t}\right\}\right],
\end{aligned}
$$

s.t.:

$$
\begin{gathered}
\rho \in \mathcal{P}, \quad \mathcal{P} \triangleq\left\{\left(\rho^{t}\right)_{t=1}^{T}: \rho_{\min }^{t} \leq \rho^{t} \leq \rho_{\max }^{t},\right. \\
\left.\frac{1}{T} \sum_{t \in \mathcal{T}} \rho^{t} \leq \rho_{\text {ave }}\right\}, \\
z_{k}^{t} \geq \sum_{t \in \mathcal{T}} c^{t}-\left(\rho^{t}-\pi_{k}^{t}\right) l^{t}, \quad z_{k}^{t} \geq 0, \quad k=1, \ldots, K,
\end{gathered}
$$


where $K$ denotes the number of random day-ahead prices in the wholesale market and $\pi_{k}^{t}$ is the realization of the day-ahead wholesale price under scenario $k$ at the time slot $t$. Additionally, $z_{k}^{t}$ and $c$ are auxiliary variables.

\subsection{Smart home model}

A smart residential home has renewable energy resources, responsive loads, and energy storage devices, which are described separately in the following.

\subsubsection{Renewable energy resource}

Every customer utilizes a renewable energy resource such as the wind or solar energy, the generation of which follows a stochastic process. Here, it is assumed that every customer can predict its renewable energy production vector $\boldsymbol{P}_{n}$ in the next operation day. For each renewable resource, we have $0 \leq P_{n}^{t} \leq P_{n}$, max , where $P_{n}^{t}$ indicates energy production from the renewable energy resource of the customer $n$ at time slot $t$, which is always smaller than the nominal capacity $P_{n, \text { max }}$.

\subsubsection{Responsive loads}

The consumption of flexible loads could be shifted to off-peak hours, when energy price is low. These loads can be supplied either by distribution grid or by the customer's own storage devices and their renewable energy resources. For every customer, $\mathcal{A}_{n}$ is the set of elastic home appliances. The consumption profile of each $a_{n} \in \mathcal{A}_{n}$ is $\boldsymbol{D}_{n, a_{n}}=\left[d_{n, a_{n}}^{1}, \ldots, d_{n, a_{n}}^{t}, \ldots, d_{n, a_{n}}^{T}\right]$, where $d_{n, a_{n}}^{t}$ is the hourly scheduled energy consumption of appliance $a$ th by EMS at time slot $t$. Here, it is assumed that every customer knows total daily energy consumption, $E_{n, a_{n}}$, of the $a$ th appliance in advance as considered in [8-12]. $E_{n, a_{n}}$ should be available during the specified time interval, $\mathcal{T}_{n, a_{n}} \triangleq\left\{\theta_{n, a_{n}}, \ldots, \varphi_{n, a_{n}}\right\}$, indicating that the appliance can perform the task on time. The value of $d_{n, a_{n}}^{t}$ is limited to the minimum and maximum levels in $t \in \mathcal{T}_{n, a_{n}}$, i.e., $d_{n, a_{n}, \min }^{t}$ and $d_{n, a_{n}, \max }^{t}$. Thus, the following constraints should be considered for each appliance:

$$
\begin{aligned}
& \sum_{t=\theta_{n, a_{n}}}^{\varphi_{n, a_{n}}} d_{n, a_{n}}^{t}=E_{n, a_{n}}, \\
& d_{n, a_{n}, \min }^{t} \leq d_{n, a_{n}}^{t} \leq d_{n, a_{n}, \max }^{t}, \quad \forall t \in \mathcal{T}_{n, a_{n}},
\end{aligned}
$$

and:

$$
d_{n, a_{n}}^{t}=0, \quad \forall t \notin \mathcal{T}_{n, a_{n}}
$$

At this stage, by introducing $\boldsymbol{D}_{n}=\left(d_{n}^{t}\right)_{t=1}^{T}$ as the energy consumption vector of elastic loads, the feasible strategy set $\mathcal{D}_{n}$ could be defined for responsive loads as:

$$
\begin{aligned}
& \mathcal{D}_{n, a} \triangleq\left\{\left(d_{n, a_{n}}^{t}\right)_{t=1}^{T} \mid \text { Constraints }(4),(5)\right\} \\
& \mathcal{D}_{n} \triangleq\left\{\left(d_{n}^{t}\right)_{t=1}^{T} \mid \boldsymbol{d}_{n}^{t}=\left(d_{n, 1}^{t}, \ldots, d_{n, A_{n}}^{t}\right), d_{n, a_{n}}^{t} \in \mathcal{D}_{n, a_{n}}\right\} .
\end{aligned}
$$

\subsubsection{Energy storage model}

In this system, storage devices of the customer $n$ are charged through distribution grid in the low-price period and discharged for supplying appliances of the customer when energy price is high. Let $b_{n}^{t}$ be the charging/discharging strategy of the customer $n$ for its energy storages at time slot $t$. When $b_{n}^{t}<0$, the storage is discharged for the internal appliances; otherwise, it is charged by the grid. In such a case, the daily scheduled vector of storage devices can be written as $\boldsymbol{B}_{n}=\left[b_{n}^{1}, \ldots, b_{n}^{t}, \ldots, b_{n}^{T}\right]$. For reliable and efficient performance of the storage devices, $b_{n}^{t}$ should lie in a specified interval,

$$
-b_{n, \max } \leq b_{n}^{t} \leq b_{n, \max }
$$

where $b_{n, \max }$ is the maximum charging/discharging rate of storage devices and $S o c_{n}^{t}$ denotes the state of charge of storage devices at the beginning of time slot $t$ that depends not only on $b_{n}^{t}$, but also on scheduling strategy in the previous time slots. $S o c_{n}^{t}$ should be smaller than maximum capacity $\left(E_{n, b \max }\right)$ of energy storage devices of customer $n$ for all the time slots expressed as:

$$
0 \leq S o c_{n}^{0}+\sum_{i=1}^{t} b_{n}^{i} \leq E_{n, b \max }, \quad \forall t \in \mathcal{T} .
$$

The assumption is that every customer knows its desired charge level at the end of the scheduling time horizon, $\operatorname{Soc}_{n}^{T}$. Therefore, the total daily energy requirement, $E_{n}^{b}$, should be provided for charging the storage devices according to Eq. (9):

$$
E_{n}^{b}=S o c_{n}^{T}-S o c_{n}^{0}, \quad \sum_{t=1}^{T} b_{n}^{t}=E_{n}^{b} .
$$

Consequently, the feasible strategy set of the storage devices is:

$$
\mathcal{B}_{n} \triangleq\left\{\left(b_{n}^{t}\right)_{t=1}^{T} \mid \text { Constraints }(7),(8),(9)\right\} .
$$

\subsubsection{Objective model of the customer}

Customer $n$ should purchase load demand $l_{n}^{t}=D_{n, i n l}^{t}+$ $b_{n}^{t}+\sum_{a_{n} \in \mathcal{A}_{n}} d_{n, a_{n}}^{t}-P_{n}^{t}$ from the grid at each time slot, where $D_{n, i n l}^{t}$ indicates inelastic loads of the consumption of the customer at time slot $t$. It is assumed that customers cannot inject energy into the grid and the load demand cannot exceed a certain value, $l_{n}^{\max }$. In this phase, the feasible strategy set of every customer is presented as: 


$$
\begin{aligned}
\overline{\mathcal{L}}_{n} \triangleq & \left\{\left(\boldsymbol{d}_{n}^{t}, b_{n}^{t}\right)_{t=1}^{T} \mid \boldsymbol{d}_{n}^{t} \in \mathcal{D}_{n}, b_{n}^{t} \in \mathcal{B}_{n},\right. \\
& \left.0 \leq l_{n}^{t} \leq l_{n}^{\max }\right\} .
\end{aligned}
$$

In this model, customers want to maximize their utility while minimizing their electrical energy bill. As previously noted, customers may collaborate in AAL adjusting program that is beneficial to both DISCO and customers. Thus, the customers, in addition to holding their individual constraints, adjust the AAL global constraint together. Hence, the objective function of each customer is formulated as:

$$
\begin{aligned}
& \left.\operatorname{Min}_{l_{n} \in \mathcal{L}_{n}} f_{n}\left(\boldsymbol{l}_{n}, \boldsymbol{\rho}\right)=\sum_{t \in \mathcal{T}} \mathbb{E}\left\{\rho^{t} l_{n}^{t}-U_{n}^{t}\right)\right\} \\
& \mathcal{L}_{n} \triangleq\left\{\left(l_{n}^{t}\right)_{t=1}^{T}: l_{n}^{t} \in \overline{\mathcal{L}}_{n}, L_{\text {ave }}^{\min } \leq L_{\text {ave }}(\boldsymbol{l}) \leq L_{\text {ave }}^{\max }\right\}
\end{aligned}
$$

where $L_{\text {ave }}^{\min }$ and $L_{\text {ave }}^{\max }$ are the lower and upper thresholds of AAL, respectively. These values should be set in a manner that the aggregate load profile becomes smoother. The AAL constraint can be broken into two constraints of $L_{\text {ave }}^{\min }-L_{\text {ave }} \leq 0$ and $L_{\text {ave }}-L_{\text {ave }}^{\max } \leq 0$, which are reduced to the form of $\boldsymbol{L}_{\text {ave }}(\boldsymbol{l}) \leq 0$.

$U_{n}^{t}$ is utility function of customer $n$ at time slot $t$. The customer satisfaction level for electricity consumption can be quantified as a utility function. In this article, the quadratic utility function is applied to modeling the preference of the customers, which has been used in $[5,10]$. This function captures the required characteristics for a utility function like non-decreasing property and non-increasing marginal benefit. This function for customer $n$ is:

$$
\begin{aligned}
U_{n}^{t}\left(\boldsymbol{d}_{n}^{t}, \omega_{n}^{t}, \kappa_{n}^{t}\right)= & \omega_{n}^{t}\left(D_{n, i n l}^{t}+\sum_{a_{n} \in \mathcal{A}_{n}} d_{n, a_{n}}^{t}\right) \\
& -\frac{\kappa_{n}^{t}}{2}\left(D_{n, i n l}^{t}+\sum_{a_{n} \in \mathcal{A}_{n}} d_{n, a_{n}}^{t}\right)^{2}
\end{aligned}
$$

where $\omega_{n}^{t}$ and $\kappa_{n}^{t}$ are the predetermined variables and vary among customers. By proper setting of these parameters, it is possible to model the value of electrical energy for each customer.

\section{Energy management game}

In this holistic energy management approach, the interactions of different agents are divided into two parts: (a) the interaction between DISCO and customers and (b) interaction of customers due to the common constraint. In the following, the proper games that capture these interactions are introduced. Also, solutions to the games and their properties are discussed.

\subsection{Stackelberg game: Interaction of DISCO and customers}

In the proposed energy management approach, first, DISCO decides on optimal day-ahead energy price by considering reaction of the customers (i.e., their consumption). Then, EMS units provide scheduling for consumption of the customers based on the received optimal price. Thus, the interaction between DISCO and customers is modeled as a Stackelberg game.

Definition 1. The Stackelberg day-ahead energy management game, $\mathbb{G}$, can be described through the tuple:

$$
\mathbb{G}=\left\{\mathcal{N} \cup\{\mathrm{DISCO}\}, \mathcal{P},\left\{\mathcal{L}_{n}\right\}_{n \in \mathcal{N}}, f_{D},\left\{f_{n}\right\}_{n} \in \mathcal{N}\right\},
$$

where:

- $\mathcal{N} \cup\{$ DISCO $\}$ is the set of players in the Stackelberg game, in which DISCO is the leader and $\mathcal{N}$ is the set of followers;

- $\mathcal{P}$ is the feasible strategy set of DISCO as defined through Eq. (3);

- $\mathcal{L}_{n}$ is the feasible strategy set for each customer as defined through Eq. (12);

- $f_{D}$ and $f_{n}$ are the objective functions of DISCO and every customer as defined through Eqs. (3) and (12), respectively.

\subsection{Non-cooperative GN game: Interaction of customers}

Every customer plays a non-cooperative GN game with other customers in response to the optimal price, because the strategy space of customers is coupled within the common constraint.

Definition 2. The non-cooperative GN game, $\overline{\mathbb{G}}$, is defined by the tuple $\overline{\mathbb{G}}=\left\{\mathcal{N},\left\{\mathcal{L}_{n}\right\}_{n \in \mathcal{N}},\left\{f_{n}\right\}_{n \in \mathcal{N}}\right\}$.

\subsection{GN and stackelberg equilibrium}

In order to compute the equilibrium of game $\overline{\mathbb{G}}$, the scheme proposed by Scutari et al. [16] is applied here. In this scheme, game $\overline{\mathbb{G}}$, is substituted with its equivalent Karush-Kuhn-Tucker (KKT) system and turned into an augmented Nash problem with $N+1$ players. DISCO acts as the $(N+1)$ th player and controls the overprice variable $\lambda$, which is the corresponding multiplier of the common constraint $\boldsymbol{L}_{\text {ave }}(\boldsymbol{l}) \leq 0$. DISCO treats the price complementary condition as an optimizing problem, $\boldsymbol{m i n}_{\boldsymbol{\lambda} \geq \mathbf{0}}-\boldsymbol{\lambda}^{\boldsymbol{T}} \boldsymbol{L}_{\boldsymbol{a v e}}(\boldsymbol{l})$. However, at this point, it acts at the same level with customers. DISCO forces the customers to meet the shared constraint by assigning $\boldsymbol{\lambda}$. In the newly extended Nash game, the objective function of $N+1$ players is expressed as: 


$$
\begin{aligned}
& \min _{l_{n} \in \overline{\mathcal{L}}_{n}}, f_{n, a u g}=f_{n}\left(\boldsymbol{l}_{n}, \boldsymbol{l}_{-n}\right)+\boldsymbol{\lambda}^{T} \boldsymbol{L}_{\text {ave }}(l), \\
& \forall n=1, \ldots, N, \\
& \min _{\boldsymbol{\lambda} \geq 0} f_{N+1}=-\boldsymbol{\lambda}^{T} \boldsymbol{L}_{\text {ave }}(\boldsymbol{l}) .
\end{aligned}
$$

In response to the optimal price vector $\rho^{*}$, which is derived from Eq. (3), every customer plays its best strategy $\boldsymbol{l}_{n}^{*}$ by considering the overprice $\boldsymbol{\lambda}^{*}$. Thus, the strategy set $\left(\boldsymbol{l}^{*}, \boldsymbol{\lambda}^{*}\right)$ is the GN equilibrium of $\overline{\mathbb{G}}$. At this point, $\operatorname{SE}\left(\boldsymbol{\rho}^{*}, \boldsymbol{l}^{*}, \lambda^{*}\right)$ occurs, which is referred to as an equilibrium solution to the proposed day-ahead energy management game.

Definition 3. In $\overline{\mathbb{G}}$, the strategy set $\left(\boldsymbol{\rho}^{*}, l^{*}, \lambda^{*}\right)$ generates SE if and only if it meets the following set of inequalities:

$$
\begin{aligned}
& f_{n, \text { aug }}\left(\boldsymbol{l}_{n}^{*}, \boldsymbol{l}_{-n}^{*}, \boldsymbol{\rho}^{*}, \boldsymbol{\lambda}^{*}\right) \leq f_{n, \text { aug }}\left(\boldsymbol{l}_{n}, \boldsymbol{l}_{-n}^{*}, \boldsymbol{\rho}^{*}, \boldsymbol{\lambda}^{*}\right), \\
& \forall n=1, \ldots, N, \\
& f_{N+1}\left(\boldsymbol{\lambda}^{*}\right) \leq f_{N+1}(\boldsymbol{\lambda}), \\
& f_{D}\left(\boldsymbol{\rho}^{*}, \boldsymbol{l}^{*}\right) \leq f_{D}\left(\boldsymbol{\rho}, \boldsymbol{l}^{*}\right),
\end{aligned}
$$

where $\boldsymbol{l}_{-n}$ is the strategy set of all customers without customer $n$.

\subsection{Existence of equilibrium and its properties} Because the optimization problem of DISCO is convex, the optimal day-ahead price can be calculated by any of the convex optimization methods [18]. A capable and proper tool, namely VI technique, is applied to study the solution and properties of the non-cooperative games [17]. However, GN game is difficult to solve and VI method is not applicable to all cases. In some situations, GN games can be reformulated as a $\operatorname{VI}(\mathcal{L}, \boldsymbol{F})$ problem [16], which finds the point $\boldsymbol{l}^{*} \in \mathcal{L}$ by meeting:

$$
\left(\boldsymbol{l}-\boldsymbol{l}^{*}\right)^{T} \boldsymbol{F}\left(\boldsymbol{l}^{*}\right) \geq 0, \quad \forall \boldsymbol{l} \in \mathcal{L} .
$$

The solution to the problem $\operatorname{VI}(\mathcal{L}, \boldsymbol{F})$ is named variational solution and computed by specifying the monotonicity property of the vector-valued function $F$. If the next lemma is satisfied, a solution to game $\overline{\mathbb{G}}$ is attained by solving its equivalent VI problem [16].

Lemma 1. In $\overline{\mathbb{G}}$, if the following conditions are met:

1. The individual strategy set $\overline{\mathcal{L}}_{n}$ is closed and convex;

2. Objective $f_{n}$ of the customers is convex and twice continuously differentiable in $l \in \mathcal{L}$ for every fixed $\boldsymbol{l}_{-n} \in \mathcal{L}_{-n}$;

3. The common constraint AAL is continuously differentiable and jointly convex $l \in \mathcal{L}$ in, then every solution to problem $\operatorname{VI}(\mathcal{L}, \boldsymbol{F})$ is a solution to the GN game (not vice versa), where $\mathcal{L} \triangleq \prod_{n=1}^{N} \mathcal{L}_{n}$ and $\boldsymbol{F}(\boldsymbol{l}) \triangleq\left(\nabla_{l_{n}} f_{n}(l)\right)_{n=1}^{N}$. For the Proof, see Appendix A.

Now that all conditions of Lemma 1 are met in game $\overline{\mathbb{G}}$, the associated $\operatorname{VI}(\mathcal{L}, \boldsymbol{F})$ problem is assessed for the variational solution to game $\overline{\mathbb{G}}$ as presented in Theorem 1.

Theorem 1. The game of the customer among EMS units, $\overline{\mathbb{G}}$, in response to the optimal price setting by DISCO has multiple Nash equilibria. For the Proof, see Appendix B.

As stated before, equilibrium of $\overline{\mathbb{G}}$ is computed through solving the equivalent augmented Nash problem (Eqs. (14) and (15)). With this approach, in the next section, we can design a distributed algorithm for converging to SE.

\section{Distributed algorithms}

In this section, we develop an algorithm for reaching the SE, which should be executed by both DISCO and customers. As proved in Theorem 1, game $\overline{\mathbb{G}}$ among EMS units has multiple equilibria. Thus, the algorithm with the best response may fail to converge here.

To address this issue, DISCO and customers in the augmented Nash game should optimize a regularized problem in every iteration of the algorithm. By regularizing the objective of the players, the monotone $\operatorname{VI}(\mathcal{L}, \boldsymbol{F})$ problem becomes strongly monotone. As a result, the game converges to one of the primal equilibria of the game. The regularized game $\overline{\mathbb{G}}$ is:

$$
\begin{aligned}
& \min _{l_{n} \in \overline{\mathcal{L}}_{n}} f_{n}+\left(\boldsymbol{\lambda}^{(i)}\right)^{T} \boldsymbol{L}_{\text {ave }}\left(\boldsymbol{l}_{n}, \boldsymbol{l}_{-n}^{(i)}\right)+\frac{\tau}{2}\left\|\boldsymbol{l}_{n}-\boldsymbol{l}_{n}^{(i)}\right\|^{2}, \\
& \forall n=1, \ldots, N \\
& \min _{\boldsymbol{\lambda} \geq 0} \boldsymbol{\lambda}^{T} \boldsymbol{L}_{\text {ave }}(\boldsymbol{l})+\frac{\tau}{2}\left\|\boldsymbol{\lambda}-\boldsymbol{\lambda}^{(i)}\right\|^{2},
\end{aligned}
$$

where $\tau$ is a regularization parameter and the superscript $i$ represents the $i$ th iteration.

The parameter $\tau$ should be chosen large enough for the convergence of the developed best-responsebased algorithm, named Proximal Decomposition Algorithm (PDA). By applying this algorithm, the customers reach one of the equilibria of $\overline{\mathbb{G}}$ without the possibility of selecting the ones that lead to uncertainty in the resulting system performance. Here, a PTRA algorithm is developed with the ability to select the desired equilibrium. The prominence of this PTRA is revealed through computation and signaling of incremental cost among customers. 


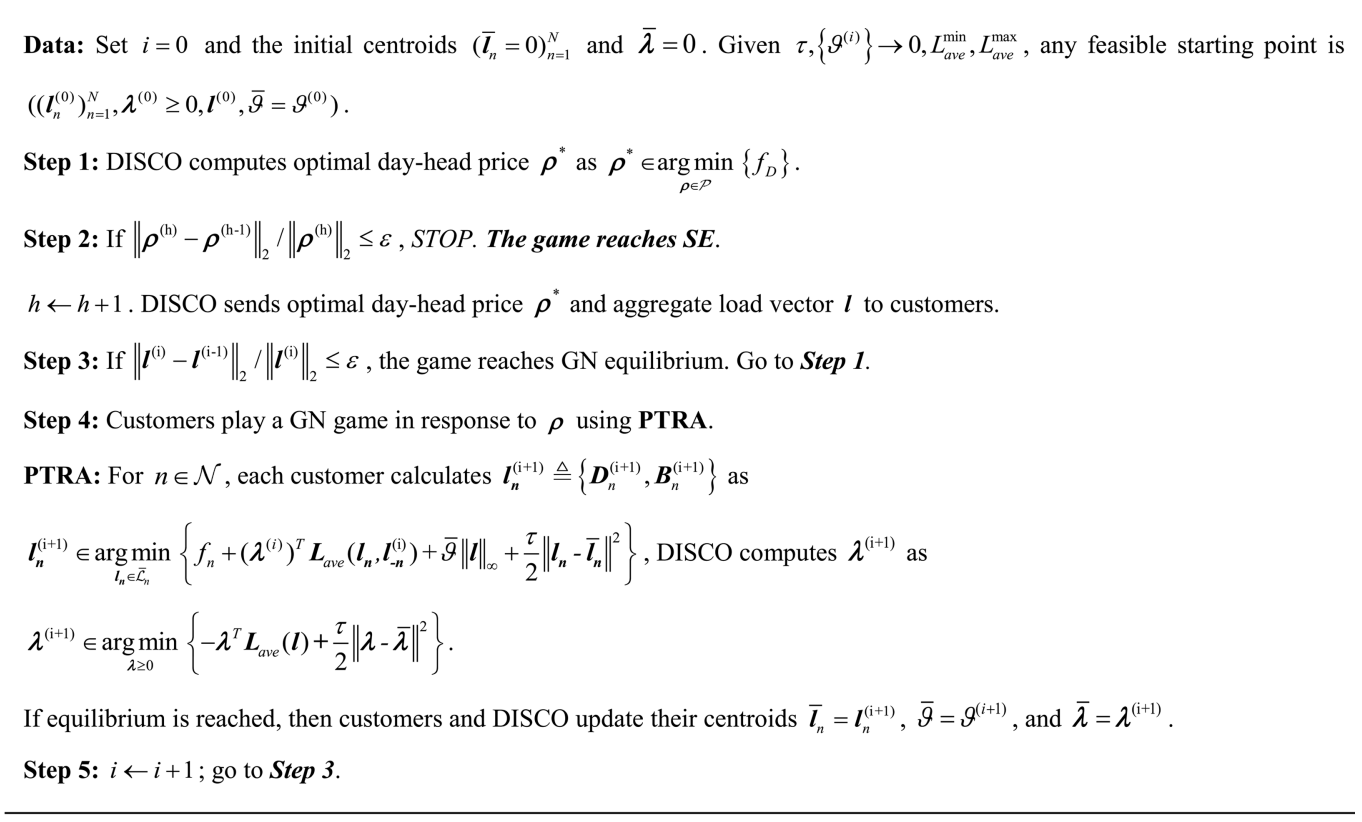

Algorithm 1: Algorithm to reach Stackelberg Equilibrium (SE).

To drive the system to the desired equilibrium, a criterion function $\phi\left(\boldsymbol{l}_{n}, \boldsymbol{l}_{-n}\right)$ should be defined aligned with the equilibrium of the interest herein. The merit function $\phi$ represents the degree of signaling and performance without which customers do not exchange any information. Otherwise, they should cooperate. According to [17, Theorem 21], $\phi$ should have the properties of being continuously differentiable and convex on $\mathcal{L}$ and having bounded level sets on the solutions to the GN game. $\nabla \phi$ is a Lipschitz continuum on $\mathcal{L}$. Accordingly, the criterion function in this algorithm is proposed as: $\phi\left(\boldsymbol{l}_{n}, \boldsymbol{l}_{-n}\right)=\|\boldsymbol{l}\|_{\infty}=$ $L_{\text {peak }}$, because it embodies all necessary conditions. In fact, customers should only agree to consider $\min \|\boldsymbol{l}\|_{\infty}$ in their optimization problem at the beginning of game $\overline{\mathbb{G}}$. With this merit function, game $\overline{\mathbb{G}}$, among the equilibria, converges to the one which reduces the maximum aggregate load. Subsequently, LF is improved. With this scheming, game $\overline{\mathbb{G}}$ converges to the considered desired equilibrium. Consequently, every customer should solve a sequence of standard regularized problems as:

$$
\begin{aligned}
\min _{l_{n} \in \overline{\mathcal{L}}} f_{n} & +\left(\boldsymbol{\lambda}^{(i)}\right)^{T} \boldsymbol{L}_{a v e}\left(\boldsymbol{l}_{n}, \boldsymbol{l}_{-n}^{(i)}\right) \\
& +\vartheta^{(i)}\|\boldsymbol{l}\|_{\infty}+\frac{\tau}{2}\left\|\boldsymbol{l}_{n}-\boldsymbol{l}_{n}^{(i)}\right\|^{2},
\end{aligned}
$$

where $\left\{\vartheta^{(i)}\right\}$ is a sequence with $\vartheta^{(i)}>0,\left\{\vartheta^{(i)}\right\} \rightarrow 0$, and $\sum_{i=0}^{\infty} \vartheta^{(i)}=\infty[17]$.

At this stage, the holistic energy management algorithm is ready to be exhibited. Initially, DISCO computes $\tau$ as given in Theorem 2 and broadcasts it to the customers. Before every playing sequence of regularized augmented Nash game, DISCO solves Eq. (3) by any convex optimization method [18] and forwards optimal day-ahead price and daily aggregate load to the customers. Then, DISCO and customers run an iterative PTRA for achieving the desired GN equilibrium. At each iteration $i$, DISCO and every customer optimize problems (20), (21), respectively. Game $\bar{G}$ ends when a termination criterion (e.g., $\left.\left\|\boldsymbol{l}^{(i)}-\boldsymbol{l}^{(i-1)}\right\|_{2} /\left\|\boldsymbol{l}^{(i)}\right\|_{2} \leq \varepsilon\right)$ is met. At this point, every customer sends its consumption profile to DISCO for the new optimal price calculating. This energy management algorithm reaches SE and ends when the calculated price does not significantly change between two consecutive iterations. The algorithm procedure and its convergence conditions are given in Theorem 2 and Algorithm 1, respectively.

Theorem 2. If in the mentioned monotone game $\overline{\mathbb{G}}$, the regularization parameter $\tau$ meets:

$$
\tau>\sqrt{N+A_{1}+\ldots+A_{N}},
$$

and $\left\{\vartheta^{(i)}\right\}$ is in the forms of $\vartheta^{(i)}>0,\left\{\vartheta^{(i)}\right\} \rightarrow 0$, and $\sum_{i=0}^{\infty} \vartheta^{(i)}=\infty$. Any sequence $\left\{\boldsymbol{d}_{n}^{(i)}, \boldsymbol{b}_{n}^{(i)}, \boldsymbol{\lambda}^{(i)}\right\}_{i=1}^{\infty}$ generated by PTRA converges to a variational solution to game $\overline{\mathbb{G}}$ and, finally, the $\mathrm{SE}$ is achieved through Algorithm 1. For the Proof, see Appendix C.

\section{Implementation of the proposed energy management mechanism}

The proposed energy management mechanism is only applicable to the smart grid platform. All of the smart 
agents of this grid should be equipped with Advanced Metering Infrastructure (AMI), sensors, controllers, and bi-directional communication system to achieve optimality in grid goals. In such context, some new technical and socio-economic challenges, which include data management, communication, cybersecurity, customer privacy, and active participation of customers in energy management program arise.

The existing large number of meters and computational units in the smart grid lead to a vast amount of data and information, which should be collected, stored, retrieved, and analyzed. Management of these big data in the smart grid is a vital issue as they may decrease the processing speed of data aggregation and analysis. Cloud-based big data analytics technology seems to be a solution to this problem. Furthermore, a proper communication protocol and media should be defined and applied to transferring data among different parties. Although wide ranges of communication technology for deployment in the smart grid exist, they have their limitations. The optical fiber has high bandwidth and low attenuation and interface, but it is very expensive and complicated.

On the other hand, cybersecurity should address information availability, integrity, and confidentiality to avoid any potential threat to system operation and privacy of the customer. In fact, cybersecurity is the other essential feature of reliable smart grid operation. Every layer of smart grid should demand specific security, which is not sufficient alone. The cybersecurity besides operation policy of operator should be in such a way that privacy of the customer is kept intact and, consequently, the customer accepts the smart grid technology. In our proposed energy management mechanism, consumption behavior of the customer and their preferences are considered as the private information of the customer and only DISCO has access to them. In other words, every customer receives only the aggregated load of the grid and the personal habits and behavior of customers are not revealed to other customers. Consequently, their privacy is preserved.

To successfully implement different operation programs in the smart grid, active participation of customers in these programs is needed. Utilities with the help of amenities and facilities along with smart grid can design different operational aspects such as tariff schemes and operational policies, which may not be accepted by the customers. In this situation, the grid operator with precise regulations and incentive policies can motivate the customers to take part actively in different operation programs or accept the new tariff scheme willingly.

\section{Simulation results}

In this section, the performance of the proposed day- ahead energy management approach by numerical simulation is evaluated. A smart distribution grid with $N=20$ customers is simulated in MATLAB software. To solve optimization problems, CVX, a package for specifying and solving convex programs, is applied [19].

Every customer has some shiftable and nonshiftable loads. Plug-in Hybrid Electric Vehicle (PHEV), washer, dryer, and dishwasher are examples of shiftable appliances that are flexible in their consumption scheduling. The second group is named non-shiftable appliances; for example TV, refrigerator, lighting, and heating have strict consumption scheduling constraints. The daily consumption of customers before management and daily inelastic demand are identified based on the model introduced in [20]. It is assumed that every customer has four shiftable appliances, as mentioned before, and their feasible operation is set based on the real consumption pattern for residential customers. For instance, PHEV owners practically charge their cars when they are at home from late night to early morning, or washing machine usually works during the day before the dryer starts to work. As stated, every customer is equipped with energy storage devices the capacity of which is random within the range of [2 $\mathrm{kWh} 3 \mathrm{kWh}]$. The initial and final charge levels of the storage devices of customers are between $\left[\begin{array}{ll}0.2 & 0.3\end{array}\right]$ and $\left[\begin{array}{ll}0.5 & 0.6\end{array}\right]$ of the storage capacity of the devices, respectively. In addition, every customer utilizes a renewable energy resource the maximum energy production of which is within the range of [ $1 \mathrm{kWh} 2 \mathrm{kWh}]$. The estimated expected wholesale price is bar charted in Figure 2.

Seven scenarios are assessed and in all of them, the customers are equipped with flexible loads and storage devices. However, only in the last four scenarios, the customers utilize renewable energy resources. In the first and fourth scenarios, no energy management is considered. In the second and fifth, every smart home benefits from PTRA energy management approach in which only storage devices with charge capability are of concern. In the third and sixth scenarios, every customer employs PTRA, but the storage devices can

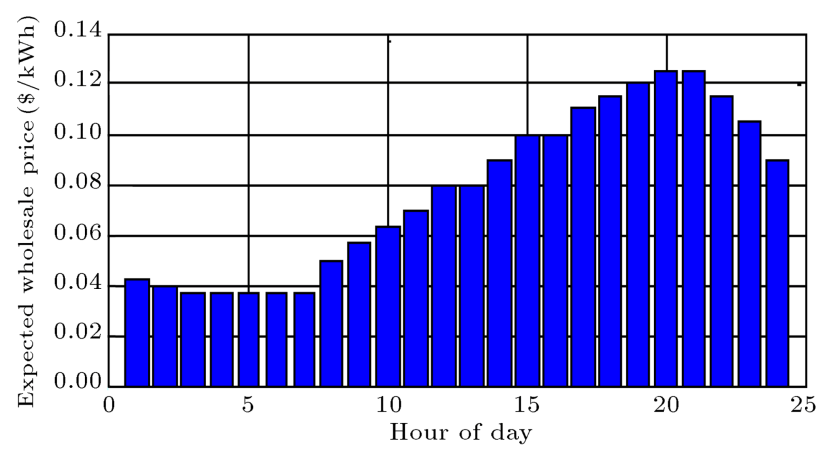

Figure 2. Expected day-ahead wholesale electricity prices. 
be charged and discharged. The condition in the last scenarios is similar to that in the sixth, but with a different management algorithm, which is PDA management approach, without predictable performance.

The aggregate load during a day through the first three scenarios is illustrated in Figure 3, in which the smoother load profile and lower maximum demand are obtained through the proposed PTRA energy management approach, indicating a greater LF and a reduction in the bill to customers. Storage devices charging in off-peak hours and discharging in peak hours in Scenario 3 further decrease the maximum aggregate load in comparison with Scenario 2.

The renewable resources, due to their stochastic nature, have variable and uncontrollable power generation. These characteristics, when applied to the distribution grid, disturb the load profile shaping and reduce LF. The aggregate demand in Scenarios 4-6 is shown in Figure 4. As observed, the PTRA approach improves LF by proper scheduling of storage devices and flexible loads. In Scenario 5, since the discharging ability of the storage devices is canceled, the peak aggregate demand is more than that in Scenario 6 .

This proposed PTRA energy management framework converges to equilibrium, which would improve the LF with a reduction in aggregate peak demand. The performances of PTRA and PDA approaches are compared in Figure 5. It is obvious that the maximum

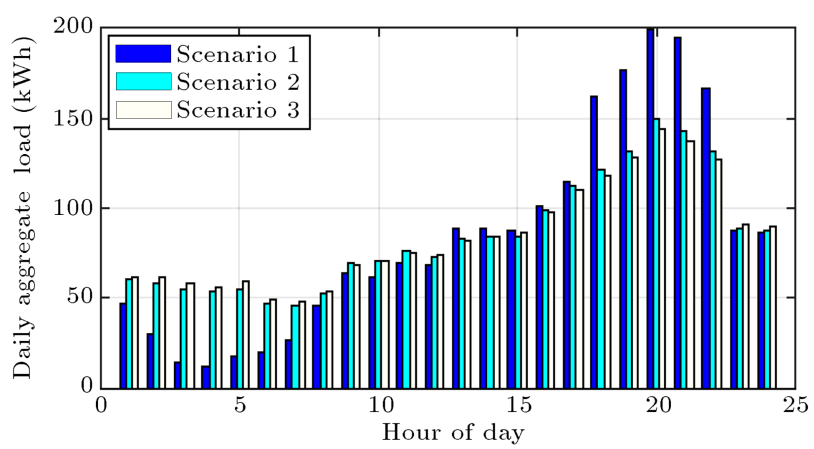

Figure 3. Daily aggregate load in the first three scenarios.

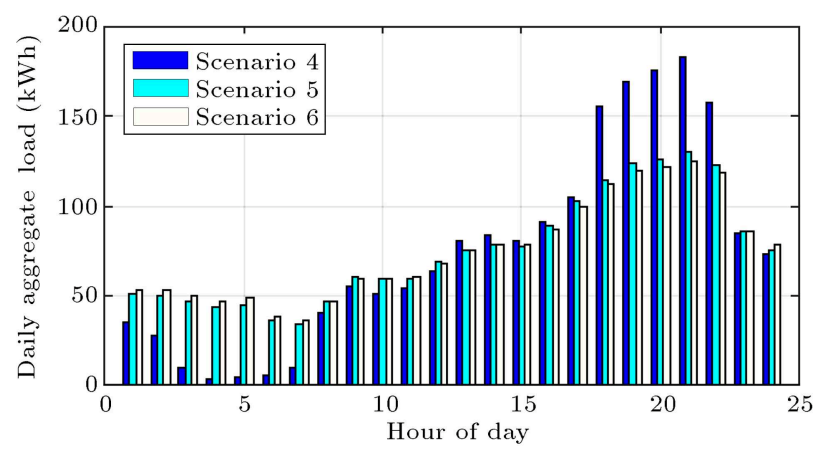

Figure 4. Daily aggregate load in the second three scenarios.

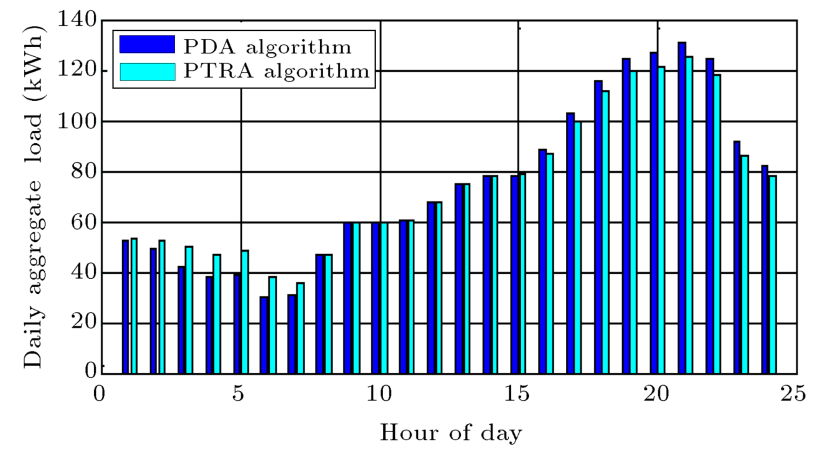

Figure 5. Daily aggregate load in Proximal Decomposition Algorithm (PDA) and Proximal Tikhonove Regularization Algorithm (PTRA).

Table 1. Load Factor (LF), maximum aggregate demand, and aggregate billing cost in the studied scenarios.

\begin{tabular}{lccc}
\hline Scenario & LF & $\begin{array}{c}\text { Maximum } \\
\text { aggregate } \\
\text { demand }(\mathbf{k W})\end{array}$ & $\begin{array}{c}\text { Aggregate } \\
\text { billing cost } \\
\mathbf{( \$ )}\end{array}$ \\
\hline Scenario 1 & 0.4267 & 198.5354 & 255.6562 \\
Scenario 2 & 0.5668 & 149.4592 & 229.2465 \\
Scenario 3 & 0.5874 & 144.2277 & 226.6945 \\
Scenario 4 & 0.4108 & 182.5725 & 232.6267 \\
Scenario 5 & 0.5765 & 130.0963 & 206.1959 \\
Scenario 6 & 0.5993 & 125.1411 & 203.6394 \\
Scenario 7 & 0.5719 & 130.8268 & 207.9699 \\
\hline
\end{tabular}

aggregate load is reduced in PTRA approach and LF increases in comparison with PDA.

The values of $\mathrm{LF}$, the maximum aggregate demand values, and the aggregate billing costs in different scenarios are tabulated in Table 1. In smart homes, which contain flexible loads and storage devices, the amount of LF becomes significantly high by utilizing the energy management program. LF increases by $24.72 \%$ and $27.35 \%$ in the second and third scenarios, respectively, in comparison with the first scenario. In a similar manner, LF increases in the presence of fluctuating renewable resources by implementing PTRA approach $(28.74 \%$ and $31.46 \%$ greater in the fifth and sixth scenarios compared with the fourth scenario), as can be seen in Table 1. The storage devices with their discharging ability contribute to the increase in LF. As expected, PTRA has higher capability than PDA in the improvement of the aggregate load shaping. This is due to equilibrium selection capability of PTRA, which is aligned with LF improvement.

As observed in Table 1, PTRA approach reduces the aggregate billing cost of customers by redistributing the elastic loads and storage scheduling in the low-price hours. The total billing cost of customers is reduced by $13 \%$ in the third scenario compared to that in the first scenario and by $14.23 \%$ in the sixth scenario compared 


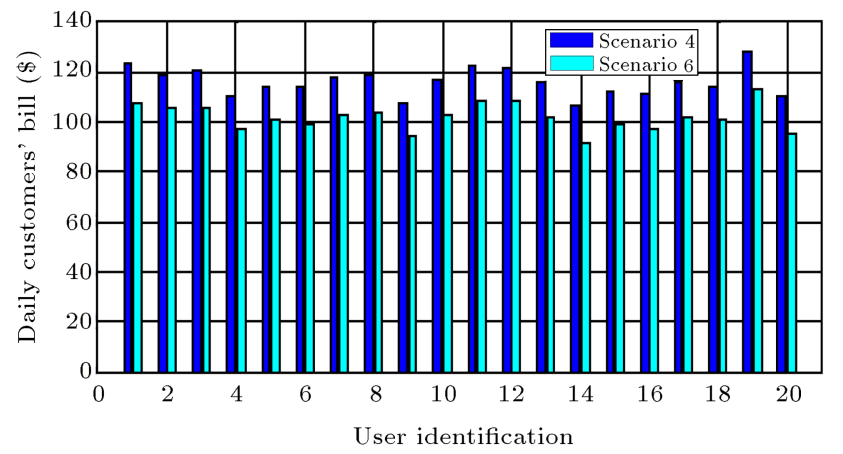

Figure 6. Daily individual bill to customers.

to that in the fourth scenario. It is obvious from Table 1 that PDA reduces the total billing cost of the system by $11.85 \%$, which is approximately $2.5 \%$ less than the reduction by PTRA.

Although PTRA decreases the aggregate billing cost of customers, an individual customer should be motivated enough to participate in PTRA management program. The daily individual bills to customers in the fourth and fifth scenarios are compared in Figure 6. It is clear that all customers pay the DISCO less and they benefit from participating in PTRA approach.

From the obtained results, it can be deduced that by proper scheduling of responsive loads and storage devices, the aggregate load shaping is improved, especially in the presence of renewable energy resources. The effect of the flexible capacity of appliances and storage devices on the aggregate billing cost and LF is shown in Figure 7. As expected, an increase in capacity enhances the aggregate load profile shape and reduces the aggregate billing cost.

\section{Conclusion}

In this paper, a holistic day-ahead distributed energy management approach in a smart distribution grid is proposed, in which both distribution company (DISCO) and its clients are involved. Energy management game and the interactions between DISCO and customers were modeled as a Stackelberg game. DISCO specified day-ahead optimal price by maximizing profit and sent it to the customers. Customers received the price and scheduled their flexible loads usage and charge/discharge storage devices profiles while meeting the common Average Aggregate Load (AAL) constraint. Consequently, the interaction among customers was modeled as a non-cooperative GN game.

In addition, customers agreed with each other to reduce the maximum aggregate load by adding a common extra term $\min \left\{\phi\left(\boldsymbol{l}_{n}, \boldsymbol{l}_{-n}\right)=\|\boldsymbol{l}\|_{\infty}=L_{\text {peak }}\right\}$ to their objectives. By adopting this approach, it was proved that the GN game converged to an equilibrium, which was in the alignment with the desired system performance (i.e., increasing Load Factor (LF)).

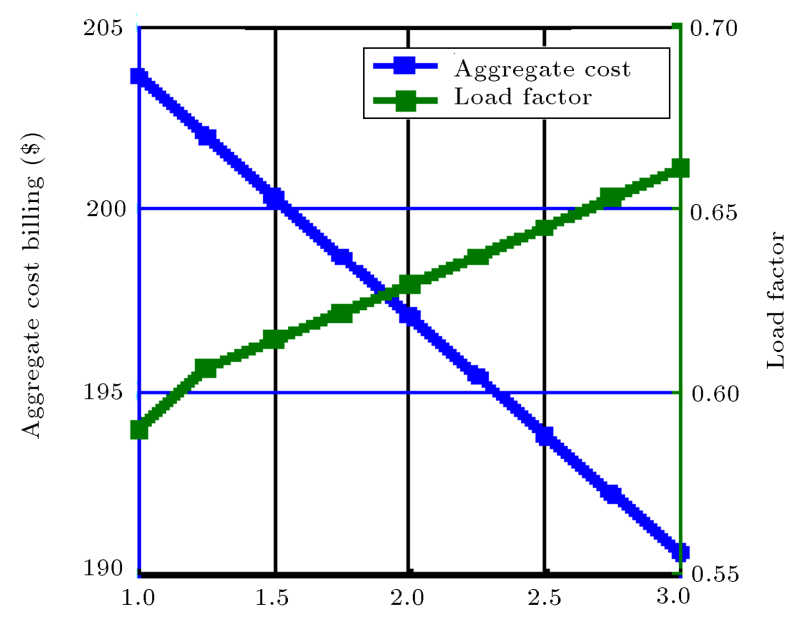

(a) Aggregate storage capacity (E/Emax)

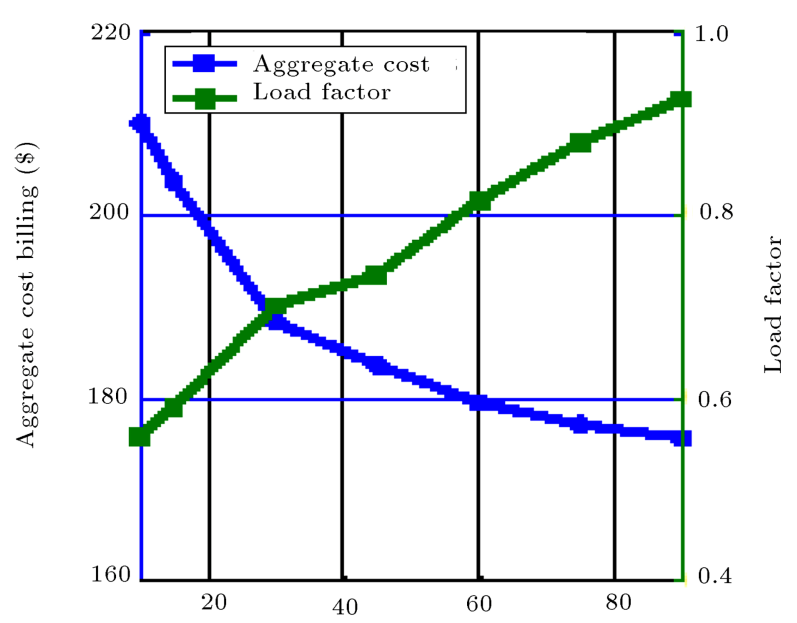

(b) Percentage of flexible load (\%)

Figure 7. Aggregate billing cost and Load Factor (LF): (a) The effect of aggregate storage capacity of devices and (b) the effect of aggregate flexible capacity of loads.

Considering both common constraint AAL and common function $\|\boldsymbol{l}\|_{\infty}$ in the customer optimization problem, the appropriate shape of the aggregate load in the presence of fluctuating production of renewable resources and without it was achieved. Consequently, an increase in the LF led to a decrease in the billing cost of customers as well as efficient facility utilization of DISCO. Numerical results verified the theoretical outcomes and illustrated that the proposed energy management approach was applicable to the practical smart grid situation.

\section{References}

1. Palensky, P. and Dietrich, D. "Demand side management: Demand response, intelligent energy systems, and smart loads", IEEE Transactions on Industrial Informatics, 7(3), pp. 381-388 (2011). 
2. Molderink, A., Bakker, V., Bosman, M.G.C., Hurink, J.L., and Smit, G.J.M. "Management and control of domestic smart grid technology", IEEE Transactions on Smart Grid, 1(2), pp. 109-119 (2010).

3. Papaefthymiou, G., Hasche, B., and Nabe, C. "Potential of heat Pumps for demand side management and wind power integration in the German electricity market", IEEE Transactions on Sustainable Energy, 3(4), pp. 636-642 (2012).

4. Yang, P., Tang, G., and Nehorai, A. "A game-theoretic approach for optimal time-of-use electricity pricing", IEEE Transactions on Power Systems, 28(2), pp. 884892 (2013).

5. Chai, B., Chen, J., Yang, Z., and Zhang, Y. "Demand response management with multiple utility companies: A two-level game approach", IEEE Transactions on Smart Grid, 5(2), pp. 722-731 (2014).

6. Atzeni, I., Ordóñez, L.G., Scutari, G., Palomar, D.P., and Fonollosa, J.R. "Demand-side management via distributed energy generation and storage optimization", IEEE Transactions on Smart Grid, 4(2), pp. 866-876 (2013).

7. Jia, L. and Tong, L. "Dynamic pricing and distributed energy management for demand response", IEEE Transactions on Smart Grid, 7(2), pp. 11281136 (2016).

8. Mohsenian-Rad, A.H., Wong, V.W.S., Jatskevich, J., Schober, R., and Leon-Garcia, A. "Autonomous demand-side Management based on game-theoretic energy consumption scheduling for the future smart grid", IEEE Transactions on Smart Grid, 1(3), pp. 320-331 (2010).

9. Chen, H., Li, Y., Louie, R.H.Y., and Vucetic, B. "Autonomous demand side management based on energy consumption scheduling and instantaneous load billing: An aggregative game approach", IEEE Transactions on Smart Grid, 5(4), pp. 1744-1754 (2014).

10. Deng, R., Yang, Z., Chen, J., Asr, N.R., and Chow, M.Y. "Residential energy consumption scheduling: A coupled-constraint game approach", IEEE Transactions on Smart Grid, 5(3), pp. 1340-1350 (2014).

11. Fadlullah, Z. M., Quan, D.M., Kato, N., and Stojmenovic, I. "GTES: An optimized game-theoretic demandside management scheme for smart grid", IEEE Systems Journal, 8(2), pp. 588-597 (2014).

12. Nguyen, H.K., Song, J.B., and Han, Z. "Distributed demand side management with energy storage in smart grid", IEEE Transactions on Parallel and Distributed Systems, 26(12), pp. 3346-3357 (2015).

13. Yaagoubi, N. and Mouftah, H.T. "User-aware game theoretic approach for demand management", IEEE Transactions on Smart Grid, 6(2), pp. 716-725 (2015).

14. Soliman, H.M. and Leon-Garcia, A. "Game-theoretic demand-side management with storage devices for the future Smart Grid", IEEE Transactions on Smart Grid, 5(3), pp. 1475-1485 (2014).
15. Maharjan, S., Zhu, Q., Zhang, Y., Gjessing, S., and Başar, T. "Demand response management in the smart grid in a large population regime", IEEE Transactions on Smart Grid, 7(1), pp. 189-199 (2016).

16. Scutari, G., Palomar, D.P., Facchinei, F., and Pang, J.S. "Monotone games for cognitive radio systems", Distributed Decision-Making and Control, ser. Lecture Notes Contr. Inf. Sci. Series, New York, NY, USA; Springer-Verlag Inc. (2011).

17. Scutari, G., Facchinei, F., Pang, J. Sh., and Palomar, D. "Real and complex monotone communication games", IEEE Trans. on Information Theory, 60(7), pp. 4197-4231 (2014).

18. Boyd, S. and Vandenberghe, L., Convex Optimization, Cambridge University Press (2009).

19. Grant, M. and Boyd, S., CVX: Matlab software for disciplined convex programming, version 2.0 beta.http://cvxr.com/cvx (2013).

20. Paatero, J.V. and Lund, P.D. "A model for generating household electricity load profiles" , Int. J. Energy Res., 30(5), pp. 273- 290 (2006).

\section{Appendix A}

\section{Proof of Lemma 1}

This lemma is based on the results obtained in $[16,17]$. The set $\overline{\mathcal{L}}_{n}$ is closed and convex, because it is polyhedra [18]. The function $f_{n}$ is convex if the Hessian matrix $H_{n}^{t} \triangleq \nabla_{l_{n}^{t}}^{2} f_{n}^{t}$ is positive semi-definite [18]. $H_{n}^{t}$ is obtained as follows:

$$
\begin{aligned}
H_{n}^{t}\left(\boldsymbol{l}^{t}\right) & =\left[\begin{array}{cc}
\frac{\partial^{2} f_{n}^{t}}{\partial \boldsymbol{d}_{n}^{t}{ }^{2}} & \frac{\partial^{2} f_{n}^{t}}{\partial b_{n}^{t} \partial \boldsymbol{d}_{n}^{t}} \\
\frac{\partial^{2} f_{n}^{t}}{\partial \boldsymbol{d}_{n}^{t} \partial b_{n}^{t}} & \frac{\partial^{2} f_{n}^{t}}{\partial b_{n}^{t}{ }^{2}}
\end{array}\right] \\
& =\left[\begin{array}{cccc}
\kappa_{n, 1}^{t} & 0 & \cdots & 0 \\
0 & \ddots & 0 & 0 \\
\vdots & 0 & \kappa_{n, A_{n}}^{t} & 0 \\
0 & 0 & 0 & 0
\end{array}\right]_{\left(A_{n}+1\right) \times\left(A_{n}+1\right)}
\end{aligned}
$$

It is clear that the eigenvalues of $H_{n}^{t}$ are non-negative, indicating that $H_{n}^{t}$ is positive semi-definite. The common constraint AAL is expressed as intersection of the two half spaces, hence having a convex set [18].

\section{Appendix B}

\section{Proof of Theorem 1}

Based on [16-Theorem 1], if $\boldsymbol{F}$ is monotone on $\mathcal{L}$, then $\operatorname{VI}(\mathcal{L}, \boldsymbol{F})$ (game $\overline{\mathbb{G}}$ ) has a convex solution set. The monotonicity property of $\boldsymbol{F}$ is realized by determining the definiteness property of Jacobian matrix [16]:

$$
J \boldsymbol{F}(\boldsymbol{l}) \triangleq\left(J_{l_{m}} \boldsymbol{F}_{n}(\boldsymbol{l})\right)_{n, m=1}^{N}
$$


which is calculated as:

$$
\begin{aligned}
J_{l_{n}^{t}} \boldsymbol{F}_{n}{ }^{t}\left(\boldsymbol{l}^{t}\right) & =H_{n}^{t}\left(\boldsymbol{l}^{t}\right) \\
J_{l_{m}^{t}} \boldsymbol{F}_{n}{ }^{t}\left(\boldsymbol{l}^{t}\right) & =\left[\begin{array}{cc}
\frac{\partial^{2} f_{n}^{t}}{\partial \boldsymbol{d}_{m}^{t} \partial \boldsymbol{d}_{n}^{t}} & \frac{\partial^{2} f_{n}^{t}}{\partial b_{m}^{t} \partial \boldsymbol{d}_{n}^{t}} \\
\frac{\partial^{2} f_{n}^{t}}{\partial \boldsymbol{d}_{m}^{t} \partial b_{n}^{t}} & \frac{\partial^{2} f_{n}^{t}}{\partial b_{m}^{t} \partial b_{n}^{t}}
\end{array}\right] \\
& =\left[\begin{array}{ccc}
0 & \cdots & 0 \\
\vdots & \ddots & \vdots \\
0 & \cdots & 0
\end{array}\right] .
\end{aligned}
$$

Here, all eigenvalues of the Jacobian matrix $J \boldsymbol{F}$ are non-negative; thus, matrix $J \boldsymbol{F}$ is positive semi-definite. Consequently, $\boldsymbol{F}$ is monotone on $\mathcal{L}$. This indicates that game $\overline{\mathbb{G}}$ has multiple equilibria and Theorem 1 is proved.

\section{Appendix C}

\section{Proof of Theorem 2}

According to $[16,17]$, the PTRA converges if the $(N+$ 1) $\times(N+1)$ matrix $\tilde{\Upsilon}_{F, \bar{v}, \boldsymbol{\tau}}$, with:

$$
\tilde{\Upsilon}_{F, \bar{\vartheta}, \tau} \triangleq\left[\begin{array}{cc}
\Upsilon_{F, \bar{\vartheta}}+\tau \mathbf{I}_{N} & -\gamma \\
-\gamma^{T} & \tau
\end{array}\right]_{(N+1) \times(N+1)},
$$

is positive definite, where:

$$
\begin{aligned}
{\left[\Upsilon_{F, \bar{\vartheta}}\right]_{n m} \triangleq \begin{cases}\alpha_{n}^{\min }, & \text { if } n=m \\
-\beta_{n m}^{\max }, & \text { if } n \neq m\end{cases} } \\
\gamma \triangleq\left(\gamma_{n}\right)_{n=1}^{N}, \gamma_{n} \triangleq \sup _{l_{n} \in \overline{\mathcal{L}}_{n}}\left\|\nabla_{l_{n}} L_{a v e}\right\|_{2}, \\
\alpha_{n}^{\min } \triangleq \inf _{l \in \overline{\mathcal{L}}} \lambda_{\min }\left(J_{l_{n}}\left(\boldsymbol{F}_{n}+\bar{\vartheta} \nabla_{l_{n}} \phi\right)\right), \\
\beta_{n m}^{\max } \triangleq \sup _{l \in \overline{\mathcal{L}}}\left\|J_{l_{m}}\left(\boldsymbol{F}_{n}+\bar{\vartheta} \nabla_{l_{n}} \phi\right)\right\| .
\end{aligned}
$$

$\lambda_{\min }\{$.$\} and \|\cdot\|$ indicate the smallest eigenvalue and the spectral norm of the matrix argument, respectively. $\tilde{\Upsilon}_{F, \bar{v}, \tau}$ is calculated as follows:

$$
\begin{aligned}
& \nabla_{l_{n}} \phi(\boldsymbol{l})= \begin{cases}{[\mathbf{1}]_{\left(\mathbf{1}+A_{n}\right)}} & t=t_{\max } \geq 0 \\
0, & \text { otherwise }\end{cases} \\
& \Rightarrow \alpha_{n}^{\min }=0, \quad \beta_{n m}^{\max }=0,
\end{aligned}
$$

$$
\begin{aligned}
& \Upsilon_{F, \bar{\vartheta}}=\left[\begin{array}{ccc}
0 & \cdots & 0 \\
\vdots & \ddots & \vdots \\
0 & \cdots & 0
\end{array}\right]_{N \times N}, \\
& \nabla_{l_{n}} \boldsymbol{L}_{\text {ave }}(\boldsymbol{l})= \begin{cases}0 & L_{\text {ave }}^{\min }-L_{\text {ave }} \leq 0 \\
\frac{1}{T}[\mathbf{1}]_{\left(1+A_{n}\right) T} & L_{\text {ave }}^{\min }-L_{\text {ave }} \geq 0 \\
0 & L_{\text {ave }}-L_{\text {ave }}^{\max } \leq 0 \\
\frac{1}{T}[\mathbf{1}]_{\left(1+A_{n}\right) T} & L_{\text {ave }}-L_{\text {ave }}^{\max } \geq 0\end{cases} \\
& \gamma_{n} \triangleq \sup _{l_{n} \in \overline{\mathcal{L}}_{n}}\left\|\nabla_{l_{n}} \boldsymbol{L}_{\text {ave }}(\boldsymbol{l})\right\|_{2} \Rightarrow \gamma_{n} \leq \sqrt{\left(1+A_{n}\right)} .
\end{aligned}
$$

Here, it can be claimed that $\bar{\Upsilon}_{F, \bar{\vartheta}, \tau} \preceq \tilde{\Upsilon}_{F, \bar{\vartheta}, \boldsymbol{\tau}}$ is true, where,

$$
\begin{aligned}
& {\left[\bar{\Upsilon}_{F, \tau}\right]_{n m}} \\
& \quad \triangleq \begin{cases}\tau & \text { if } n=m \\
0 & \text { if } n \neq m \text { and } n, m \neq N+1 \\
-\sqrt{\left(1+A_{n}\right)} & \text { otherwise }\end{cases}
\end{aligned}
$$

It is clear that $\bar{\Upsilon}_{F, \tau}$ is positive definite if the condition $\tau>\sqrt{N+A_{1}+\ldots+A_{N}}$ is satisfied.

\section{Biographies}

Azin Shojaei Berjouei received the MSc degree in Electrical Engineering from Isfahan University of Technology (IUT), Isfahan, Iran, in 2009. She is currently working toward the $\mathrm{PhD}$ degree in Algorithmic Energy Management in the Smart Grid at IUT. Her main research interests are smart grid, power quality, and integration of distributed resources.

Mehdi Moallem (SM'90) received the $\mathrm{PhD}$ degree in Electrical Engineering from Purdue University, West Lafayette, IN, USA, in 1989. He is currently a Full Professor with the Department of Electrical and Computer Engineering at Isfahan University of Technology (IUT), Isfahan, Iran. He has authored more than 150 peer-reviewed journal and conference papers. His research interests include the design and optimization of electromagnetic devices; application of advanced numerical techniques and expert systems to the analysis, diagnosis, and design of electrical machines; and power quality. Dr. Moallem is the recipient of many international and national awards.

Mohammad Hossein Manshaei received the BSc degree in Electrical Engineering and the MSc degree in Communication Engineering from Isfahan University of Technology (IUT) in 1997 and 2000, respectively. He earned another MSc degree in Computer Science and his PhD in Computer Science and Distributed Systems 
from the University of Nice Sophia-Antipolis, France, in 2002 and 2005, respectively. He did his dissertation at INRIA, Sophia Antipolis, France. He is an Assistant Professor at IUT, Iran. From 2006 to 2011, he was a senior researcher and lecturer at EPFL, Switzerland.
He was a visiting researcher at the University of North Carolina at Charlotte (UNCC) during summer 2013. His research interests include wireless networking, wireless security and privacy, social networks, cognitive radios, and game theory. 